\title{
Many-body effects due to the electron-electron interaction in silicene under an applied exchange field: The case of valley-spin coupling
}

\author{
Cite as: J. Appl. Phys. 127, 054305 (2020); doi: 10.1063/1.51 16786 \\ Submitted: 27 June 2019 . Accepted: 20 January 2020 . \\ Published Online: 5 February 2020 \\ M. Mirzaei, ${ }^{7}$ T. Vazifehshenas, ${ }^{1, a)}$ (D) T. Salavati-fard, ${ }^{2,3}$ M. Farmanbar, ${ }^{4}$ and B. Tanatar $^{5}$ (D)

\begin{abstract}
AFFILIATIONS
${ }^{1}$ Department of Physics, Shahid Beheshti University, G. C., Evin, Tehran 1983969411, Iran

${ }^{2}$ Department of Physics and Astronomy, University of Delaware, Newark, Delaware 19716, USA

${ }^{3}$ Department of Chemical and Biomolecular Engineering, University of Houston, Houston, Texas 77204, USA

${ }^{4}$ Faculty of Science and Technology and MESA+ Institute for Nanotechnology, University of Twente, P.O. Box 217, 7500 AE Enschede, The Netherlands

${ }^{5}$ Department of Physics, Bilkent University, Bilkent, 06800 Ankara, Turkey
\end{abstract}

a) Author to whom correspondence should be addressed: t-vazifeh@sbu.ac.ir

\begin{abstract}
We investigate the many-body effects induced by the electron-electron interaction in a valley-spin-polarized silicene under a perpendicularly applied exchange field. We calculate the real and imaginary parts of the self-energy within the leading order dynamical screening approximation where the screened interaction is obtained from the random phase approximation. Our study on the valley- and spin-dependent real and imaginary parts of the self-energy indicates that the different coupled valley-spin subbands may exhibit distinct characteristics. Moreover, we obtain the corresponding spectral functions and find that the plasmaron and quasiparticle peaks have different spectral weights and broadenings in all states. Interestingly, it seems that there are clear dependencies for the position and broadening of the peaks on valley-spin indexes. In addition, we study the effect of the electron-electron interaction on the renormalized velocity in the on-shell approximation and show that the renormalized velocity in gapped states becomes greater, and in gapless states, it becomes smaller as the wave vector grows.
\end{abstract}

Published under license by AIP Publishing. https://doi.org/10.1063/1.5116786

\section{INTRODUCTION}

Since the discovery of graphene in $2004,{ }^{1}$ there have been considerable theoretical and experimental studies on two-dimensional (2D) materials, such as transition metal dichalcogenides, black phosphorus, silicene, and topological insulators. ${ }^{2-10}$ Among these are group IV materials characterized by a low-buckled honeycomb structure with gapped Dirac points. Silicene ${ }^{11}$ (Fig. 1), the second element of this group, has been synthesized on different substrates such as $\operatorname{Ag}(111),{ }^{12} \operatorname{Ir}(111),{ }^{13}$ and $\mathrm{ZrB}_{2}(0001) .{ }^{14}$ In the band structure of monolayer silicene, minima of the conduction band and maxima of the valence band are located at the corner of the hexagonal Brillouin zone (Dirac points) with a gap induced by the intrinsic spin-orbit coupling (SOC) of about $7.8 \mathrm{meV} .{ }^{15}$ It is worth pointing out that the SOC plays an important role in transport properties of a wide range of $2 \mathrm{D}$ systems. ${ }^{16}$

The intrinsic SOC makes silicene even more promising than graphene ${ }^{22}$ as the SOC-induced bandgap in silicene is tunable by a perpendicular electric field, $E_{z}{ }^{23}$ The applied electric field, on the other hand, breaks the inversion symmetry of the lattice due to the buckling. It also opens up the degenerate spin-subbands of inequivalent valleys, which can be controlled by an exchange field through the Zeeman effect. ${ }^{24}$ The spin and valley degrees of freedom contribute to different interesting phenomena, such as the spin Hall effect (SHE) and the valley Hall effect (VHE), and can be employed to manipulate the electronic systems. ${ }^{25-29}$ The fact that the spin and valley degrees of freedom can be coupled makes such systems significant for potential applications. Silicene as a compatible 


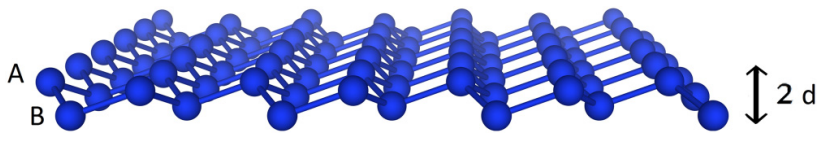

FIG. 1. Schematic side view of the buckled structure of monolayer silicene. The $A$ and $B$ sites are separated by a vertical distance $2 d$.

2D material with Si-based electronics has attracted increasingly attention especially, after introducing the first silicene-based field effect transistor that works at room temperature. ${ }^{30}$ On the other hand, the many-body effects that are important in determining the electronic and optical properties of $2 \mathrm{D}$ materials have been experimentally investigated. ${ }^{31,32}$ Angle resolved photoemission spectroscopy (ARPES) that produces in-depth information on the electronic properties of $2 \mathrm{D}$ atomic layers can be used as a probe for the manybody effects in such systems. ${ }^{33}$ Furthermore, it was shown that STM measurements can be used to provide insights into many-body interactions at the atomic scale. ${ }^{34}$ Both of these experimental techniques have been widely used to quantify characteristics of silicene on various substrates. ${ }^{33,35-37}$ Therefore, it is worthwhile to investigate the dynamical many-body properties of silicene, thoroughly.

Self-energy, a complex function that its imaginary and real parts determine the damping and energy of the quasiparticles, respectively, plays a central role in the quantum mechanical manybody theories. All important interaction effects may be included in the self-energy function. It is a difficult job to calculate the selfenergy exactly; as a result, some approximations have been introduced for obtaining the self-energy of an interacting system. It is fair to recognize the $\mathrm{G}^{0} \mathrm{~W}$ approximation as a successful approach for calculating the self-energy of a Fermi gas system with the longrange Coulomb interaction. ${ }^{38}$ Nevertheless, it should be pointed out that it fails in some cases. ${ }^{39}$ One of the important many-body properties of the system that could be obtained from the selfenergy is the single-particle spectral function $A(\mathbf{k}, \omega)$, a key quantity in characterizing the density of electronic states as a function of both momentum and energy. The spectral function can be measured by the ARPES experiments and gives useful information about the electronic structure of the system such as the full quasiparticle energy dispersion. ${ }^{40,41}$ There has been many theoretical works on the electronic properties of silicene; ${ }^{24,42-45}$ however, a comprehensive study on the self-energy of silicene under simultaneously applied external fields is absent.

Spin- and valley-dependent carrier properties in silicene can be controlled by the external electric and exchange fields. For this reason, silicene is a promising candidate for spintronic and valleytronic applications. Applying external fields interestingly reveals the spin- and valley-polarized particle-hole and plasmon excitations. ${ }^{24,42}$ Optical conductivity measurements can be used as a sensitive means to demonstrate the signatures of spin- and valley-dependent properties of silicene. ${ }^{46,47}$

Motivated by the significance of interactions, enhanced by externally applied fields, in the transport and optical properties of the system, we calculate the zero-temperature self-energy of an $\mathrm{n}$-type doped silicene including the electron-electron interaction in the presence of the perpendicular electric and exchange fields within the $\mathrm{G}^{0} \mathrm{~W}$ approximation. The effective interaction is given by the dynamical random phase approximation (RPA). This approach has been used successfully for studying the many-body effects in several systems such as $3 \mathrm{D}$ and $2 \mathrm{D}$ electron gas systems ${ }^{48,49}$ and also monolayer and bilayer graphene. ${ }^{50,51}$ Particularly, we are interested in the valley-spin polarized metal (VSPM) phase of silicene due to its rich underlying valley and spin physics. ${ }^{24,52}$ We show that both the real and imaginary parts of silicene self-energy depend upon the spin and valley degrees of freedom where the change in the imaginary part is more pronounced. Moreover, we obtain the single-particle spectral function of carriers for four different states (four distinct combinations of the spin and valley indexes). The observed peaks in the spectral function are attributed to the quasiparticle and plasmaron excitations. Plasmaron is a collective mode consisting of the plasmon and a hole. Recently, the plasmaron has been observed in graphene using ARPES measurements. ${ }^{53}$ We show that existence of the quasiparticle and plasmaron peaks in the wave vector smaller than the Fermi wave vector depends on the spin and valley indices and a chosen substrate, similar to the cases of the $3 \mathrm{D}$ and 2D electron gas systems and monolayer and bilayer graphene. ${ }^{48,50,54-56}$ Interestingly, we find that the spectral function has a sharp plasmaron peak in two states and broadened peaks in the others. Furthermore, we investigate the effect of electron-electron interaction on the valley and spin-dependent renormalized velocity, a quantity that can be directly measured from the quasiparticle dispersion. Following the method that was successfully used in the case of graphene, ${ }^{54}$ we compute the renormalized velocity and compare it with the bare electron velocity of different subbands. Our results on the many-body effects in VSP silicene may be useful to better interpret the spin-valley-resolved experimental data.

The rest of the paper is organized as follows. Section II presents the low-energy Hamiltonian of silicene and its eigenvalues in the presence of both external electric and exchange fields. The formalism and results of calculations for the self-energy, spectral function, and renormalized velocity of VSP silicene are given in Sec. III. Finally, concluding remarks of this study are outlined in Sec. IV.

\section{LOW-ENERGY HAMILTONIAN}

We consider a single-layer silicene composed of two sublattices (A and $\mathrm{B}$ ) of silicon atoms separated by a vertical distance of $2 d=0.46 \AA$ under perpendicularly applied electric and magnetic fields.

Carriers in such a system can be described by the following effective tight-binding Hamiltonian: ${ }^{26,57}$

$$
\begin{aligned}
H= & -t \sum_{\langle i, j\rangle, s} c_{i, s}^{\dagger} c_{j, s}+i \frac{\Delta_{s o}}{3 \sqrt{3}} \sum_{\langle\langle i, j\rangle\rangle, s, s^{\prime}} v_{i j} c_{i, s}^{\dagger} \sigma_{z}^{s, s^{\prime}} c_{j, s^{\prime}} \\
& -d \sum_{i, s} \varsigma_{i} E_{z}^{i} c_{i, s}^{\dagger} c_{i, s}+\sum_{i, s} M_{i} c_{i, s}^{\dagger} \sigma_{z}^{s s} c_{i, s}
\end{aligned}
$$

where in the first term, sum runs over all nearest-neighbor pairs $(\langle i, j\rangle)$ with $t=1.61 \mathrm{eV} .^{57} c_{i, s}^{\dagger}\left(c_{i, s}\right)$ is the creation (annihilation) operator at site $i$ with spin $s$. The second term includes the 
intrinsic SOC with $\Delta_{s o}=3.9 \mathrm{meV}^{15,27}$ (the effect of Rashba-SOC is ignored), where $\sigma_{i}(i=x, y, z)$ are the Pauli spin matrices and $v_{i, j}=\left(\vec{d}_{i} \times \vec{d}_{j}\right) . \hat{z} /\left|\left(\vec{d}_{i} \times \vec{d}_{j}\right) . \hat{z}\right|$, with $\vec{d}_{i}$ and $\vec{d}_{j}$ connecting the nextnearest neighbors. Moreover, $\langle\langle i, j\rangle\rangle$ denotes a sum over all those pairs. ${ }^{58}$ The third term is the on-site potential difference $\left(\Delta_{z}=E_{z} d\right)$ between two sublattices, ${ }^{59}$ and $E_{z}$ is the perpendicular electric field with $\varsigma_{i}=+1(-1)$ for $A(B)$ sites. The last term includes the interactions due to the exchange field, $M$, which is induced by the ferromagnetic substrate or adatoms. In graphene deposited on the EuO substrate, the exchange field is predicted to be about $5 \mathrm{meV} .{ }^{60}$ In order to show the effects more clearly, we use a larger value for this field, as was used previously by Van Duppen et al. ${ }^{24}$

The Hamiltonian given in Eq. (1) near the Dirac points and for low-energy electrons can be written in the spin- and valleydependent continuum model as ${ }^{15}$

$$
H_{\eta s_{z}}=v_{F}\left(\eta k_{x} \tau_{x}+k_{y} \tau_{y}\right)+\eta s_{z} \Delta_{s o} \tau_{z}-\Delta_{z} \tau_{z}+M s_{z}
$$

In this equation, $\eta= \pm 1$ denotes $\mathrm{K}_{1}$ and $\mathrm{K}_{2}$ valleys, $s_{z}= \pm 1$ shows the spin states, $\tau_{i}(i=x, y, z)$ are Pauli matrices in the sublattice space, and $v_{F}=5 \times 10^{5} \mathrm{~m} / \mathrm{s}^{59}$ It should be pointed out that we use $\hbar=1$ throughout this paper.

The energy spectrum of the Hamiltonian in Eq. (2) corresponding to the conduction $(+)$ and valence $(-)$ bands is given as

$$
E_{\eta s_{z}}(k)= \pm \sqrt{\left(v_{F} k\right)^{2}+\left(\Delta_{z}-\eta s_{z} \Delta_{s o}\right)^{2}}+s_{z} M
$$

As a result, the electronic structure of silicene, when both the electric and magnetic fields are applied perpendicularly, contains spin-split subbands in each valley with spin- and valley-dependent gap, which is twice the $\Delta_{\eta s_{z}}, 2 \Delta_{\eta s_{z}}=2\left|\Delta_{z}-\eta s_{z} \Delta_{s o}\right|$. This is actually a feature of silicene that allows $E_{z}$ to control the spin-split bandgaps. Depending on the magnitude of $E_{z}$, silicene can be found in different phases. It has been shown that the system transforms from a topological insulator (TI) to a band insulator (BI) if $\Delta_{z}$ goes from a value smaller than $\Delta_{s o}$ to a larger one. ${ }^{23,59}$ Among this phase transition, there is a critical value for the applied electric field with $\Delta_{z}=\Delta_{s o}$. This special case introduces the important VSPM phase. ${ }^{61}$ In the VSPM phase, one of the subbands experiences no gap and the other a gap of $2 \Delta_{s o}$. In other words, one of the bandgaps becomes minimized, while the other reaches its maximum value. ${ }^{24}$ Without exchange field, the spin-split subbands in the two valleys are equal but with reversed spin labels. The exchange field, on the other hand, alters the subband density of states in each valley. ${ }^{24}$ Figure 2 shows all spin- and valleydependent subbands of the VSPM phase. Here, the green solid line displays the position of Fermi energy, $\mu_{0}$, with respect to the subbands, and the horizontal axis represents the dimensionless $2 \mathrm{D}$ wave vector, $k a$, with $a$ being the lattice constant $a=3.86 \AA$. The interesting VSPM regime is the focus of this paper. By choosing $\Delta_{z}=\Delta_{s o}$ and $M \neq 0$, we study some spin- and valley-dependent many-body properties of silicene.
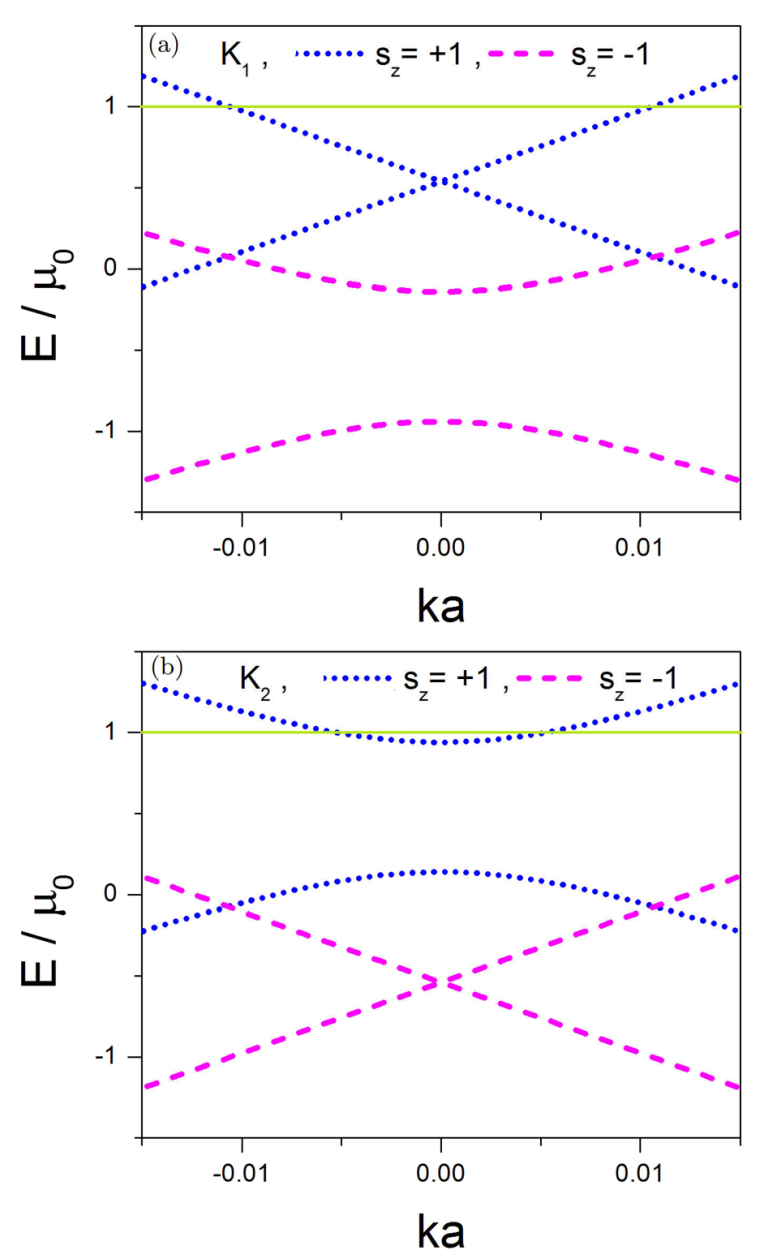

FIG. 2. Low-energy spectrum of VSP silicene under an external exchange field around two valleys (a) $K_{1}$ and (b) $K_{2}$ as a function of $k$. The blue dotted and red dashed lines correspond to the spin-up and spin-down states, respectively. The green solid line shows the Fermi energy, $\mu_{0}$. Here, we set $\Delta_{z}=\Delta_{s 0}$, $M=2.7 \Delta_{\text {so }}$, and $\mu_{0}=5 \Delta_{s o}$.

\section{MANY-BODY FORMULATION AND RESULTS}

Many-body systems in condensed matter physics are the main subject of research. In such systems, investigating the different interactions that are responsible for the fascinating behaviors is of great interest. Many theoretical and experimental efforts have been made to understand the macroscopic and microscopic properties of matter. Electronic and optical properties of a system may strongly depend on the presence of different interactions such as coupling to the substrate, electron-impurity scattering, and electron-phonon interaction. When the electron Fermi energy is comparable to the phonon energies, the electron-phonon interaction becomes significant. Since the Fermi energy of carriers in silicene can be controlled by external fields, 
it is quite possible to choose the Fermi energy far from the phonons' energy range. In this paper, we consider only the electron-electron interaction and can neglect the electron-phonon interaction because the selected Fermi energy is sufficiently lower than the optical phonon energy in silicene. Also, due to the weak bonds in silicene, the deformation potential constant is small, resulting in a weak electron-acoustic phonon coupling. ${ }^{62,63}$ The electron-electron Coulomb interaction that governs the manybody properties of the electron gas systems can be described by the following Hamiltonian: ${ }^{64}$

$$
H_{\text {int }}=\frac{1}{2 S} \sum_{q \neq 0} V(q)\left(\mathbf{n}_{\mathbf{q}} \mathbf{n}_{-\mathbf{q}}-\mathbf{N}\right)
$$

where $V(q)=2 \pi e^{2} / \kappa q$ ( $\kappa$ being the background dielectric constant) is the bare Coulomb interaction, $S$ is the area of the sample, $\mathbf{n}_{\mathbf{q}}$ is the electron density operator, and $\mathbf{N}$ is the number operator.

\section{A. Self-energy}

As mentioned earlier, the self-energy accounts for the electronic interactions and plays a crucial role in determining the complex energy of quasiparticles. In this section, we calculate the real and imaginary parts of the silicene dynamical self-energy function, considering their spin and valley dependencies in the presence of the externally applied electric and magnetic fields. We employ the $\mathrm{G}^{0} \mathrm{~W}$ approximation corresponding to the firstorder perturbation expansion in the dynamically screened Coulomb interaction, as shown in Fig. 3, in which the screening is included by the RPA dynamical dielectric function. Using the self-energy, the effect of the interaction on some physical quantities such as the quasiparticle lifetime within the on-shell approximation, spectral function, renormalized velocity, and effective mass ${ }^{38,48,52,65}$ can be calculated. The self-energy within the $\mathrm{G}^{0} \mathrm{~W}$ approximation for band $\lambda$ and subband $\left(\eta, s_{z}\right)$ is given by ${ }^{38}$

$$
\begin{aligned}
\Sigma_{\lambda \eta s_{z}}\left(\mathbf{k}, i \omega_{n}\right)= & \frac{-1}{\beta} \sum_{\lambda^{\prime}} \int \frac{d^{2} \mathbf{q}}{(2 \pi)^{2}} F_{\lambda \lambda^{\prime} \eta s_{z}}(\mathbf{k}, \mathbf{k}+\mathbf{q}) \\
& \times \sum_{m=-\infty}^{+\infty} W\left(\mathbf{q}, i \Omega_{m}\right) G_{\lambda^{\prime} \eta s_{z}}^{0}\left(\mathbf{k}+\mathbf{q}, i \omega_{n}+i \Omega_{m}\right),
\end{aligned}
$$

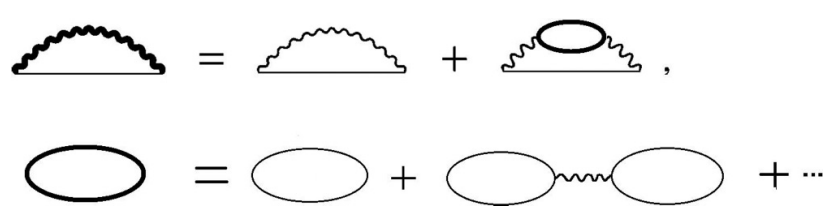

FIG. 3. Feynman diagrams for the electronic self-energy within the $\mathrm{G}^{0} \mathrm{~W}-\mathrm{RPA}$. The thin (thick) solid line indicates the noninteracting (interacting) electron Green's function, and the thin and thick wavy lines show the bare and screened Coulomb potential, respectively. where $\beta=1 / k_{B} T, F_{\lambda \lambda^{\prime} \eta s_{z}}(\mathbf{k}, \mathbf{k}+\mathbf{q})$ is the overlap function and given by ${ }^{66}$

$$
F_{\lambda \lambda^{\prime} \eta s_{z}}(\mathbf{k}, \mathbf{k}+\mathbf{q})=\frac{1}{2}\left(1+\lambda \lambda^{\prime} \frac{v_{F}^{2} \mathbf{k} \cdot(\mathbf{k}+\mathbf{q})+\Delta_{\eta s_{z}}^{2}}{\Lambda_{\eta s_{z}}(\mathbf{k}) \Lambda_{\eta s_{z}}(\mathbf{k}+\mathbf{q})}\right)
$$

with $\Lambda_{\eta s_{z}}(\mathbf{k})=\sqrt{\left(v_{F} \mathbf{k}\right)^{2}+\Delta_{\eta s_{z}}^{2}} \cdot \omega_{n}$ and $\Omega_{m}$ are the Matsubara fermion and boson frequencies and $G^{0}\left(\mathbf{k}, i \omega_{n}\right)$ is the noninteracting Green's function, which is given by

$$
G_{\lambda \eta s_{z}}^{0}\left(\mathbf{k}, i \omega_{n}\right)=\frac{1}{i \omega_{n}-E_{\eta s_{z}}(k)+\mu_{0}} .
$$

The dynamical screened effective interaction $W\left(\mathbf{q}, i \Omega_{m}\right)$ $=V(q) / \epsilon\left(\mathbf{q}, i \Omega_{m}\right)$ is determined by the bare Coulomb potential, $V(q)=2 \pi e^{2} / \kappa q$, and the dynamical dielectric function $\epsilon\left(\mathbf{q}, i \Omega_{m}\right)$, which within the RPA can be obtained from the following relation:

$$
\epsilon\left(\mathbf{q}, i \Omega_{m}\right)=1-V(q) \Pi^{0}\left(\mathbf{q}, i \Omega_{m}\right)
$$

In the above equation, $\Pi^{0}\left(\mathbf{q}, i \Omega_{m}\right)$ is the noninteracting polarization function. ${ }^{38}$ Assuming negligible transitions between different spin and valley states, the dynamical polarization function, in Eq. (8), can be expressed by summation over the spin and valley degrees of freedom as $\Pi^{0}(\mathbf{q}, i \Omega)=\sum_{\eta= \pm 1} \sum_{s_{z}= \pm 1} \Pi_{\eta s_{z}}^{0}(\mathbf{q}, i \Omega),{ }^{24}$ with $^{66}$

$$
\begin{aligned}
\Pi_{\eta s_{z}}^{0}(\mathbf{q}, i \Omega)= & \int \frac{d^{2} \mathbf{k}}{(2 \pi)^{2}} \sum_{\lambda \lambda^{\prime}} F_{\lambda \lambda^{\prime} \eta s_{z}}(\mathbf{k}, \mathbf{k}+\mathbf{q}) \\
& \times \frac{n_{F}\left(\lambda \Lambda_{\eta s_{z}}(\mathbf{k})\right)-n_{F}\left(\lambda^{\prime} \Lambda_{\eta s_{z}}(\mathbf{k}+\mathbf{q})\right)}{i \Omega+\lambda \Lambda_{\eta s_{z}}(\mathbf{k})-\lambda^{\prime} \Lambda_{\eta s_{z}}(\mathbf{k}+\mathbf{q})},
\end{aligned}
$$

where $n_{F}\left(\lambda \Lambda_{\eta s_{z}}(\mathbf{k})\right)$ is the Fermi-Dirac distribution function with the spin-dependent Fermi level, $\mu_{s}=\mu_{0}-s_{z} M$.

The retarded self-energy is obtained from Eq. (5) via analytic continuation, $i \omega \rightarrow \omega+i \eta$, after performing the Matsubara frequency summation $\left(\sum_{m}\right)$. At zero temperature, the retarded self-energy can be written as a sum of two terms, line and residue in the form of $\Sigma_{\lambda \eta s}^{r e t}(\mathbf{k}, \omega)=\Sigma_{\lambda \eta s_{s}}^{l i n e}(\mathbf{k}, \omega)+\Sigma_{\lambda \eta s}^{r e s}(\mathbf{k}, \omega)$, which are given as ${ }^{38,55,67}$

$$
\begin{aligned}
\Sigma_{\lambda \eta s_{z}}^{l i n e}(\mathbf{k}, \omega)= & -\sum_{\lambda^{\prime}} \int \frac{d^{2} \mathbf{q}}{(2 \pi)^{2}} \int_{-\infty}^{+\infty} \frac{d \Omega}{2 \pi} \frac{V(q)}{\epsilon(\mathbf{q}, i \Omega)} \\
& \times \frac{F_{\lambda \lambda^{\prime} \eta s_{z}}(\mathbf{k}, \mathbf{k}+\mathbf{q})}{i \Omega+\omega-\xi_{\lambda, \eta s_{z}}(\mathbf{k}+\mathbf{q})}
\end{aligned}
$$



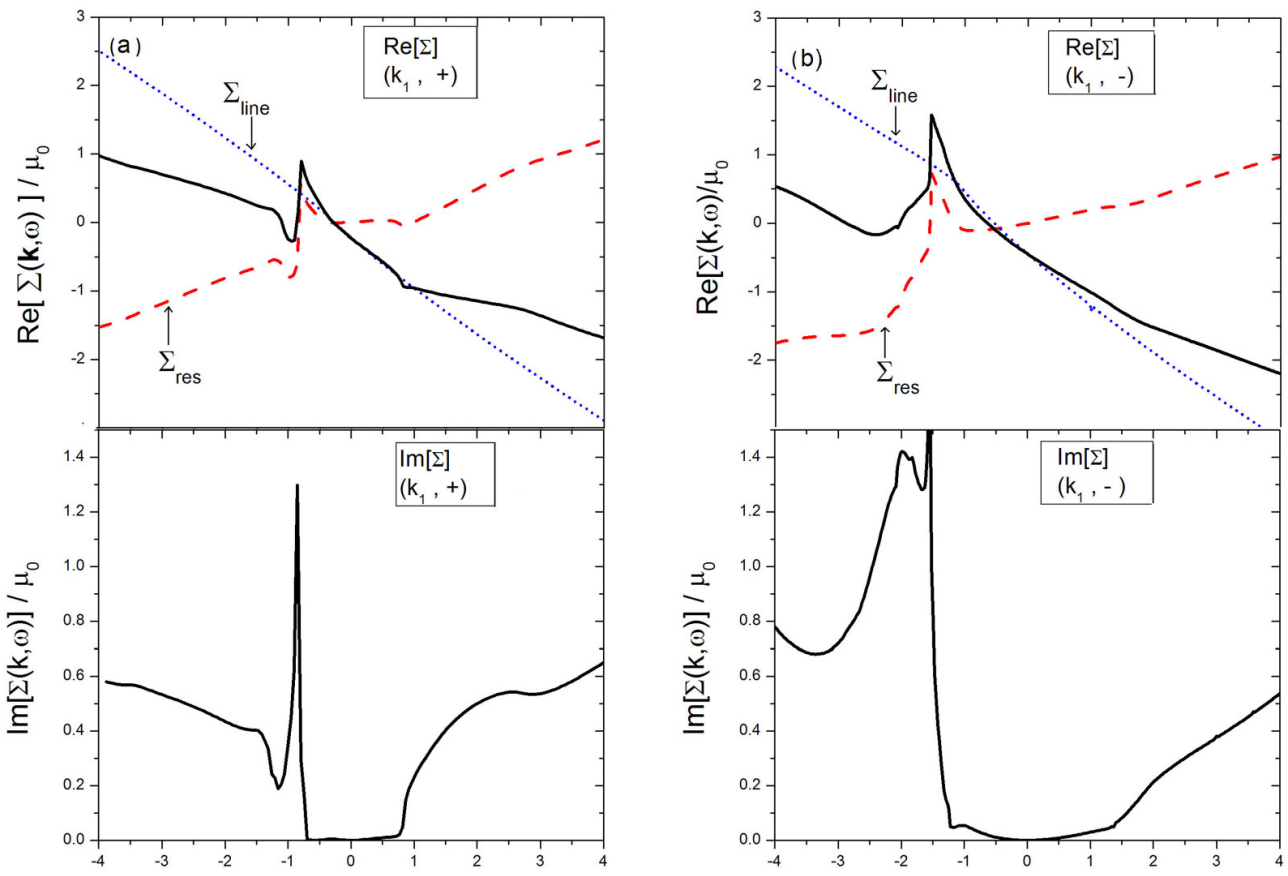

$\omega / \mu_{0}$

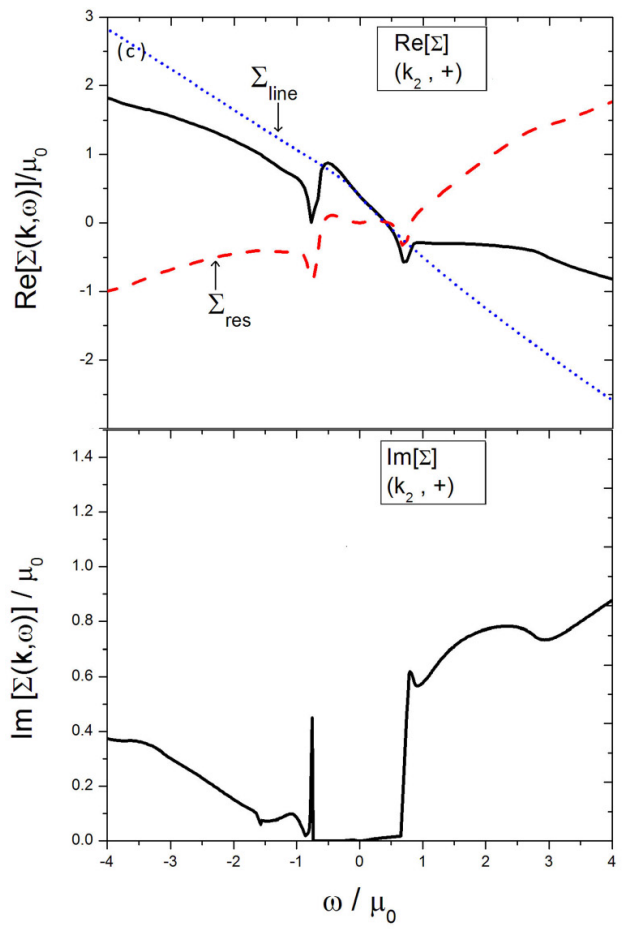

$\omega / \mu_{0}$

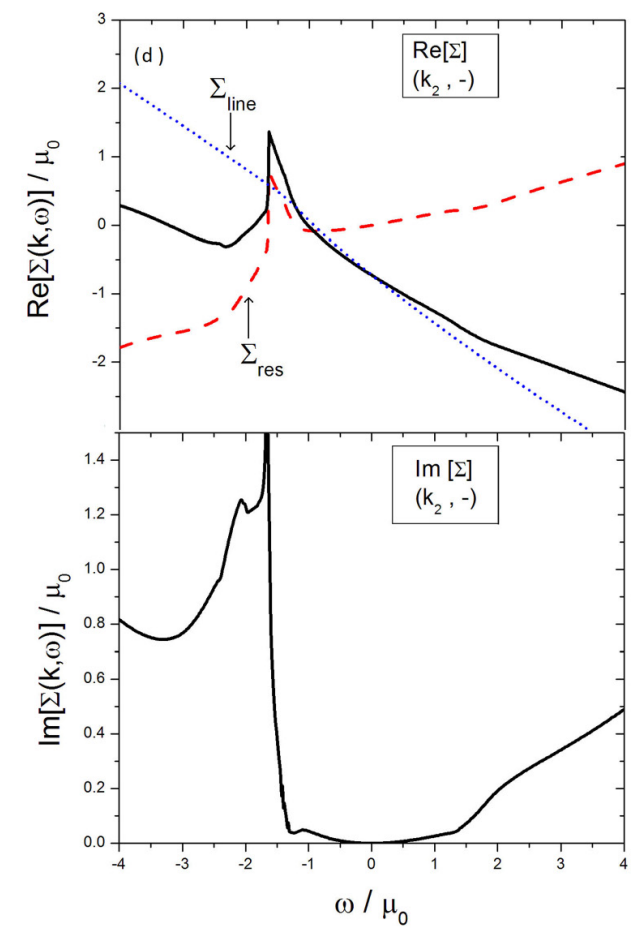

FIG. 4. Real (top panels) and imaginary (bottom panels) parts of the spin- and valley-dependent self-energy $\Sigma(k, \omega)$ in units of $\mu_{0}$ as a function of energy for $k=0.25 \mathrm{k}_{F}$ in (a) $\left(K_{1},+\right)$, (b) $\left(K_{1},-\right)$, (c) $\left(K_{2},+\right)$, and (d) $\left(K_{2},-\right)$ states of an $n$-doped VSP silicene. Here, we set $\Delta_{z}=\Delta_{s o}, M=2.7 \Delta_{s o}$, and $\mu_{0}=5 \Delta_{s 0}$. 
and

$$
\begin{aligned}
\Sigma_{\lambda \eta s_{z}}^{r e s}(\mathbf{k}, \omega)= & \sum_{\lambda^{\prime}} \int \frac{d^{2} \mathbf{q}}{(2 \pi)^{2}} \frac{V(q) F_{\lambda \lambda^{\prime} \eta s_{z}}(\mathbf{k}, \mathbf{k}+\mathbf{q})}{\epsilon\left(\mathbf{q}, \omega-\xi_{\lambda, \eta s_{z}}(\mathbf{k}+\mathbf{q})\right)} \\
& \times\left[\Theta\left(\omega-\xi_{\lambda \lambda_{1} \eta s_{z}}(\mathbf{k}+\mathbf{q})\right)-\Theta\left(-\xi_{\lambda \eta_{1} \eta s_{z}}(\mathbf{k}+\mathbf{q})\right)\right]
\end{aligned}
$$

where $\xi_{\lambda \eta s_{z}}=\lambda \Lambda_{\eta s_{z}}-\mu_{s}$ is the free particle energy and $\Theta(x)$ is the Heaviside step function. The line contribution to the selfenergy is entirely real; however, the residue term is a complex
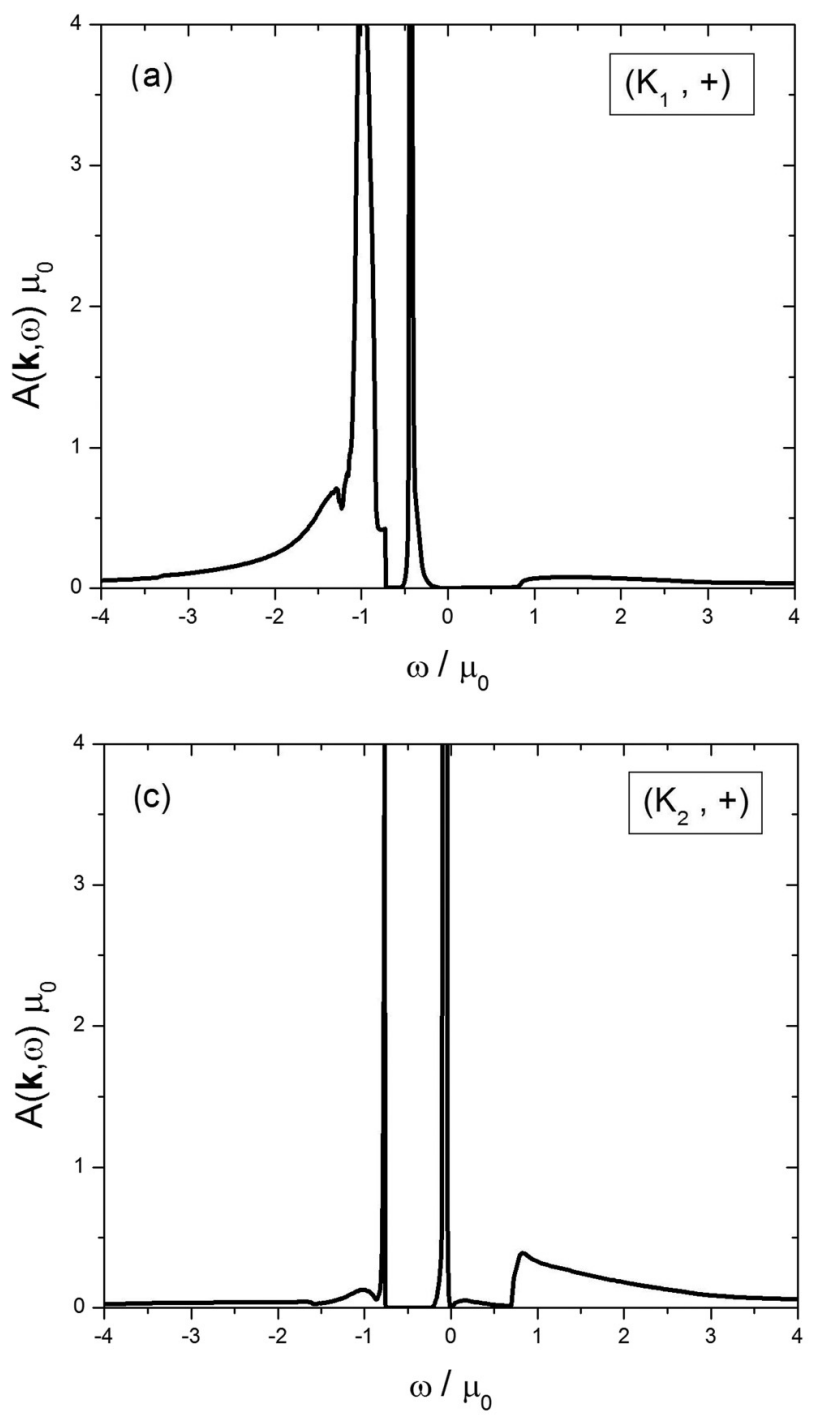

function. In Fig. 4, we plot the real and imaginary parts of the dynamical self-energy of silicene in different spin-valley states, when the electric and magnetic fields are perpendicularly applied. Here, we consider $E_{z}=\Delta_{s o}, M=2.7 \Delta_{s o}{ }^{24} \mu_{0}=5 \Delta_{s o}$, $k=0.25 k_{F}$, which characterizes the quasiparticles with a wave vector below the Fermi surface. Also, we choose $\mathrm{SiO}_{2}$ as a silicene substrate $(\kappa=2.5)$ that is commonly used in $2 \mathrm{D}$ structures with a honeycomb lattice such as graphene and $\mathrm{MoS}_{2}$.

There are two major scattering mechanisms that determine the overall structure of the self-energy: excitations of plasmons and particle-hole pairs. The line contribution is a smooth curve in all
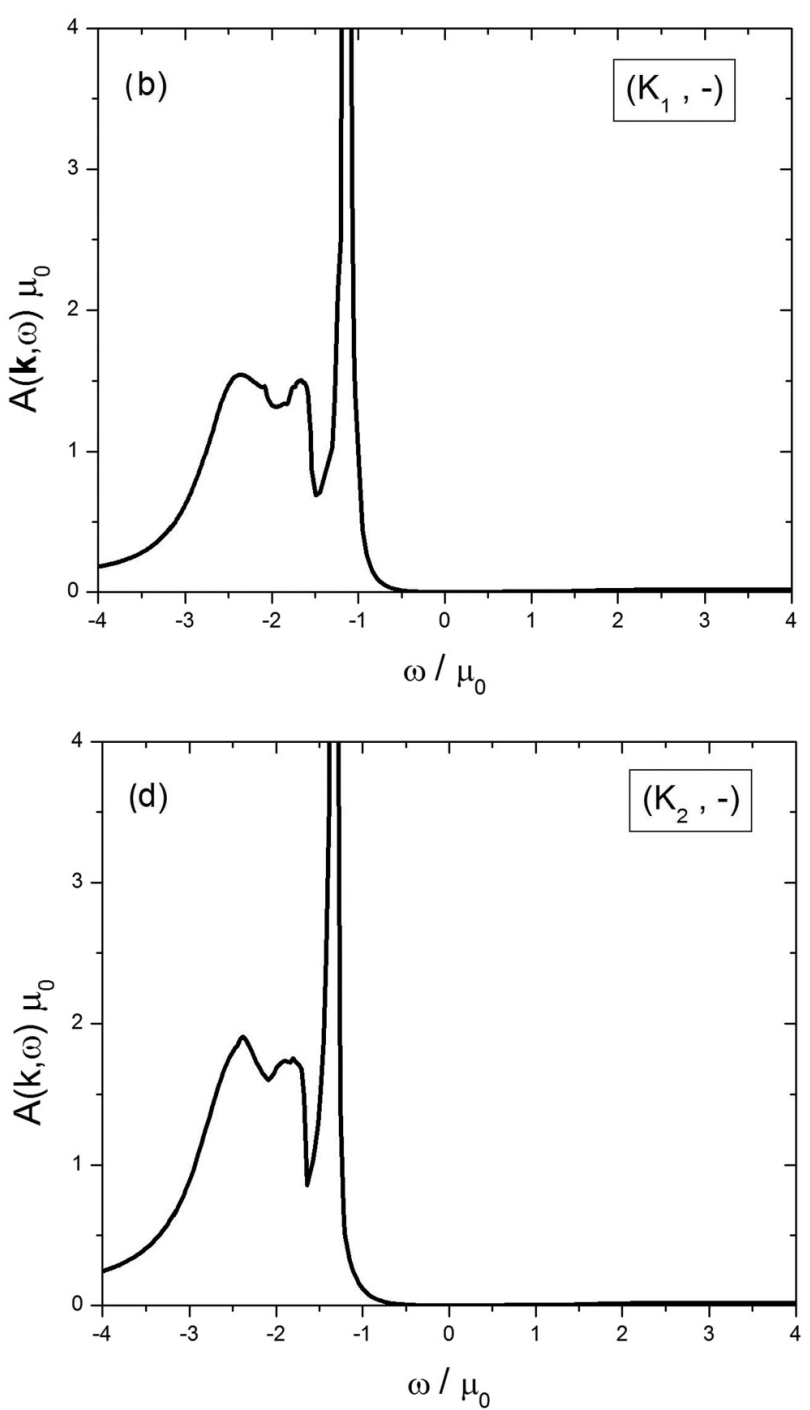

FIG. 5. Valley- and spin-dependent spectral function in (a) $\left(\mathrm{K}_{1},+\right)$, (b) $\left(\mathrm{K}_{1},-\right),(\mathrm{c})\left(\mathrm{K}_{2},+\right)$, and (d) $\left(\mathrm{K}_{2},-\right)$ states of an n-type doped VSP silicene as a function of energy for $k=0.25 \mathrm{k}_{\mathrm{F}}$. The dominant peaks are associated with the quasiparticle and plasmaron peaks. Here, we set $\Delta_{z}=\Delta_{\mathrm{so}}, M=2.7 \Delta_{\mathrm{so}}, \mu_{0}=5 \Delta_{\mathrm{so}}$, and $\mathrm{SiO} \mathrm{O}_{2}$ as a substrate with $\kappa \approx 2.5$. 
spin-valley states, as shown in Fig. 4. Moreover, the imaginary part of the self-energy displays logarithmic singularities, which come from plasmon contributions. There is a corresponding dip in the real part, $\operatorname{Re} \Sigma$, through the Kramers-Kronig relation between $\operatorname{Re} \Sigma$ and $\operatorname{Im} \Sigma$.

Below the Fermi energy, the intraband single-particle excitations contribute to $\operatorname{Im} \Sigma$, and above the Fermi energy, it is the interband excitations that enhance $\operatorname{Im} \Sigma$. Contributions from these two excitations depend on the density of carriers in the conduction band and also the gap size. ${ }^{67}$

According to this figure, there are some differences between the self-energy results of the diverse states with different electron densities.
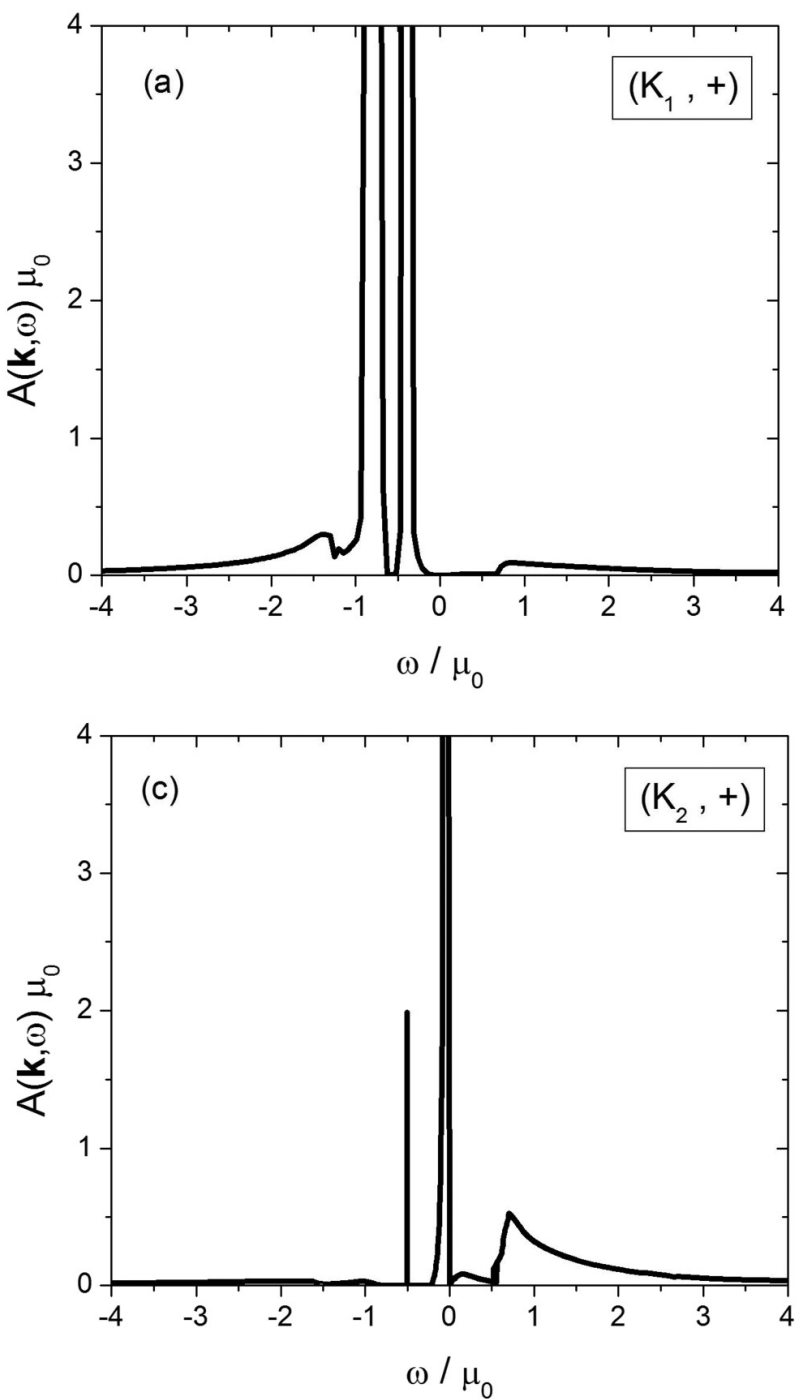

In particular, the imaginary part of the self-energy illustrates a pronounced variation with the valley and spin indexes. Also, we can see that a kink in the real part of the self-energy in a gapless $\left(\mathrm{K}_{1},+\right)$ state and a gapped $\left(\mathrm{K}_{2},+\right)$ state at $\omega \simeq \mu_{0}$ disappears in $\left(\mathrm{K}_{2},-\right)$ and $\left(\mathrm{K}_{1},-\right)$ states, which have higher electron densities, similar to the graphene ${ }^{65}$ and $2 \mathrm{D}$ electron gas ${ }^{48}$ cases, respectively.

\section{B. Spectral function}

The spectral function $A(\mathbf{k}, \omega)$ gives the probability of finding a particle (quasiparticle) in the system with momentum $\mathbf{k}$ and energy

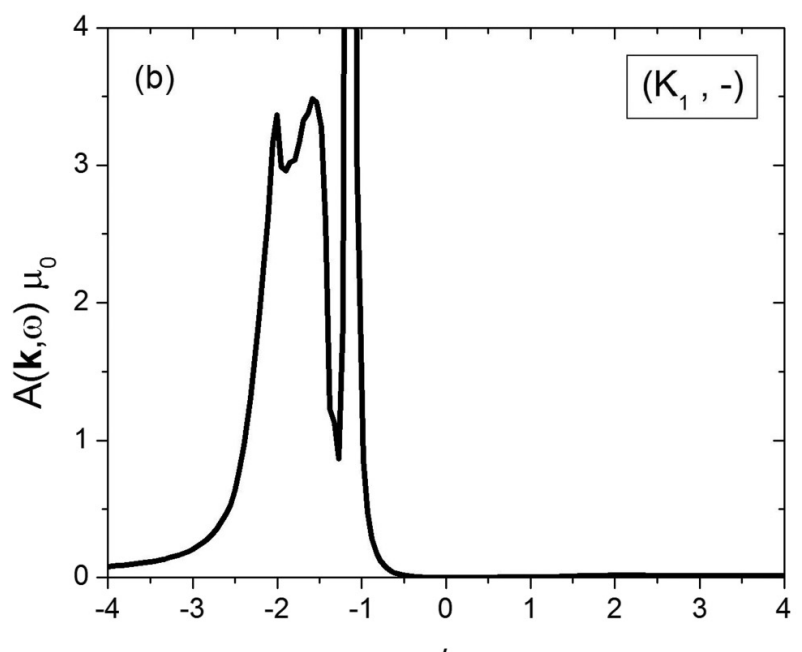

$\omega / \mu_{0}$

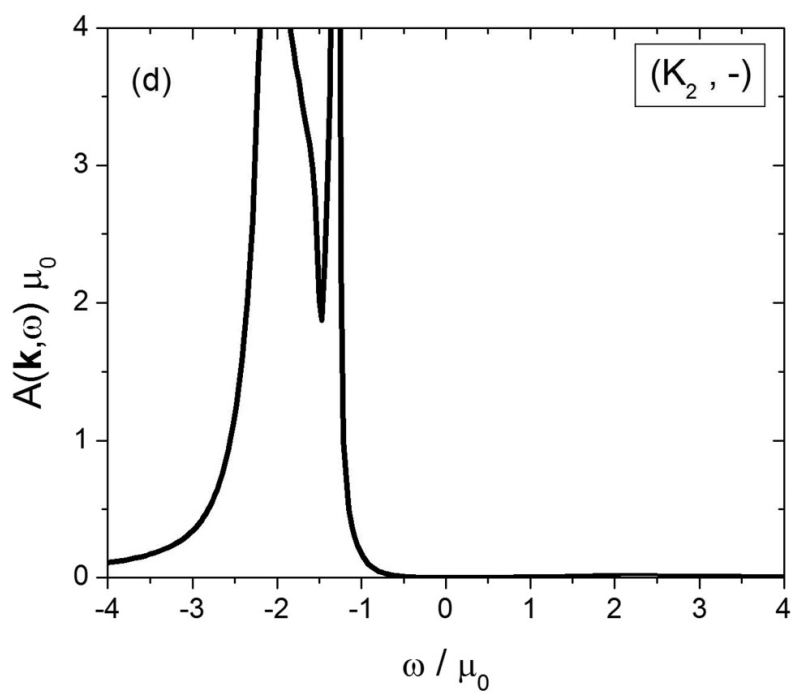

FIG. 6. Valley- and spin-dependent spectral function in (a) $\left(K_{1},+\right)$, (b) $\left(K_{1},-\right)$, (c) $\left(K_{2},+\right)$, and $(d)\left(K_{2},-\right)$ states of an n-type doped VSP silicene as a function of energy for $k=0.25 \mathrm{~K}_{\mathrm{F}}$. The dominant peaks are associated with the quasiparticle and plasmaron peaks. Here, we set $\Delta_{\mathrm{z}}=\Delta_{\mathrm{so}}, M=2.7 \Delta_{\mathrm{so}}, \mu_{0}=5 \Delta_{\mathrm{so}}$, and $\mathrm{SiC}$ as a substrate with $\kappa \approx 5.3$. 
$\omega$. This quantity contains significant information about the dynamical structure of the system. The spectral function is obtained from the self-energy through the following equation: ${ }^{68}$

$$
A_{\lambda \eta s_{z}}(\mathbf{k}, \omega)=\frac{2\left|\operatorname{Im} \Sigma_{\lambda \eta s_{z}}(\mathbf{k}, \omega)\right|}{\left[\omega-\xi_{k \eta s_{z}}-\operatorname{Re} \delta \Sigma_{\lambda \eta s_{z}}(\mathbf{k}, \omega)\right]^{2}+\left[\operatorname{Im} \Sigma_{\lambda \eta s_{z}}(\mathbf{k}, \omega)\right]^{2}},
$$

where $\delta \Sigma_{\lambda \eta s_{z}}(\mathbf{k}, \omega)=\Sigma_{\lambda \eta s_{z}}(\mathbf{k}, \omega)-\Sigma_{\lambda \eta s_{z}}\left(k_{F}, 0\right)$ and the energy of the noninteracting electrons measured from the chemical potential, $\xi_{k n s_{z}}=E_{\lambda \eta s_{z}}-\mu_{0}{ }^{68}$ As is clear from Eq. (12), the spectral function is a Lorentzian function in which the peak position is determined by $\operatorname{Re} \Sigma$ and $|\operatorname{Im} \Sigma|$ represents the linewidth. In the absence of interactions, the spectral function is a Dirac delta function $A^{0}(\mathbf{k}, \omega)=\delta(\omega-\xi(\mathbf{k}))$, while the interactions broaden the $\delta$-function peak where $\operatorname{Im} \Sigma \neq 0$. We find that the spectral function of VSP silicene has two peaks within the RPA [see Figs. 5(a)-5(d) showing the spectral function in different spin-valley states]. The first peak (going from $\omega=0$ toward negative frequencies) corresponds to quasiparticles, which are bare particles surrounded by a cloud of virtual plasmon and particle-hole excitations. Moreover, silicene spectral function has a plasmaron peak, i.e., a peak associated with the charged particle and real plasmon excitations. ${ }^{48,68}$

Intersections of $\operatorname{Re} \Sigma$ with $\omega-\xi_{k}$ indicate the quasiparticle and plasmaron peaks, which are solutions of Dyson's equation. ${ }^{48}$ By comparing the obtained spin- and valley-dependent spectral functions of silicene, we can see the effect of different electron densities of subbands on the results. It should be noted that the plasmaron and quasiparticle peaks have different spectral weights and broadening in four spin-split subbands. The broadening of the plasmaron peak is more evident in $\left(\mathrm{K}_{1},-\right)$ and $\left(\mathrm{K}_{2},-\right)$ states rather than $\left(\mathrm{K}_{1},+\right)$ and $\left(K_{2},+\right)$. This is due to the higher electron densities in the spin-down states with respect to the spin-up ones according to Fig. 2. Thus, it is expected that the electron density that can be tuned by the electric and exchange fields plays an important role in determining the behavior of a subband spectral function in all phases of silicene. Carrier density dependence of the spectral function has been obtained in the cases of $2 \mathrm{D}$ electron gas and bilayer graphene. ${ }^{48,50}$ It is worth noting that the broad peak characterizes the short lifetime of plasmaron excitations. As it can be observed from Fig. 5, the plasmaron peak has a slightly different position in each distinct state.

One of the factors that can tune the electron-electron interaction is using a different substrate. Different substrates modify $\kappa$, the background dielectric constant, in Eq. (4). To show this effect, we plot all spin-split subband spectral functions of silicene on $\mathrm{SiC}$ in Fig. 6. Except for the dielectric constant, the other parameters are the same as those in Fig. 5. As the substrate changes from $\mathrm{SiO}_{2}$ (Fig. 5) to $\mathrm{SiC}$ (Fig. 6), the dielectric screening will be increased and the electron-electron interaction undergoes some changes. The main effect of changing the substrate from $\mathrm{SiO}_{2}$ to $\mathrm{SiC}$ is increasing magnitude of the plasmaron peak in the $K_{1}$ and $K_{2}$ subband with spin down, and destroying the plasmaron peak in $\left(K_{2},+\right)$. Moreover, the difference between spectral functions of distinct subbands becomes more pronounced by using substrates with higher $\kappa$. In the absence of the exchange field, $M$, since densities of carriers in $\left(K_{1},+\right)$ and $\left(K_{2},-\right)$ are identical and also in $\left(K_{1},-\right)$ with $\left(K_{2},+\right)$, they will show the same behavior. Applying the exchange field destroys the observed symmetry between subbands and changes the Fermi energy of each state. As a result, four possible states show different responses in the spectral function.

\section{Renormalized velocity}

An important quantity that is significantly influenced by the interactions is the renormalized velocity. Frequency and wave vector dependent renormalized velocity is obtained by taking the wave vector derivative of the quasiparticle energy according to the following relation:

$$
v_{\eta s_{z}}^{\star}(k)=\frac{\partial \varepsilon_{\eta s_{z}}(k)}{\partial k} .
$$

The modified energy dispersion of electrons due to the electronelectron interaction is given by the solution to Dyson's equation for a given $\mathbf{k}$,

$$
\varepsilon_{\eta s_{z}}(\mathbf{k})=\xi_{\mathbf{k} \eta s_{z}}+\left.\operatorname{Re} \Sigma_{\eta s_{z}}(\mathbf{k}, \omega)\right|_{\omega=\varepsilon_{\eta \xi_{z}}(\mathbf{k})} .
$$

In the on-shell approximation $\omega=\xi_{\mathbf{k} \eta s_{z}}$, the renormalized velocity can be obtained as

$$
v_{\eta s_{z}}^{\star}(k)=\frac{v_{F}^{2} k}{\sqrt{\left(v_{F} k\right)^{2}+\Delta_{\eta s_{z}}^{2}}}+\frac{d}{d k} \operatorname{Re} \Sigma_{\eta s_{z}}\left(k, \xi_{k \eta s_{z}}\right) .
$$

In Fig. 7, we demonstrate the renormalized velocity, $v_{\eta s_{2}}^{*}(k)$, calculated within the on-shell approximation in units of the bare Fermi velocity as a function of wave vector in a VSP silicene under

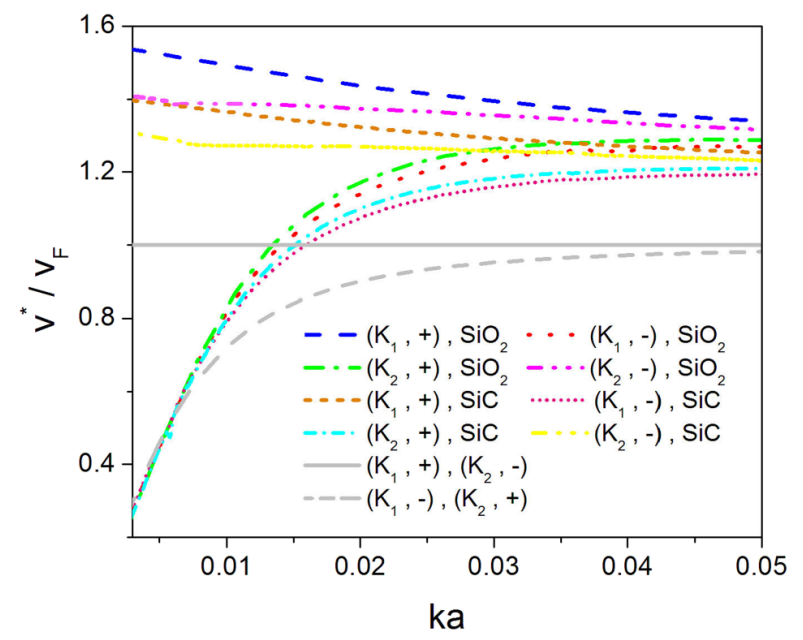

FIG. 7. The renormalized velocity in four spin-split subbands as a function of dimensionless wave vector ka within on-shell approximation. $\mathrm{SiC}$ and $\mathrm{SiO}_{2}$ are considered as substrates with $\kappa \simeq 5.3$ and 2.5 , respectively. The black lines show the velocity without electron-electron interaction correction in four subbands. Here, we set $\Delta_{z}=\Delta_{s o}, M=2.7 \Delta_{s o}$, and $\mu=5 \Delta_{s o}$. 
an external exchange field. Furthermore, we investigate the effects of the substrate on the renormalized velocity by obtaining the results of two different substrates, $\mathrm{SiO}_{2}$ and $\mathrm{SiC}$. As seen in Fig. 7 , the renormalized velocity reduces by increasing the background dielectric constant as a result of a stronger screening effect. For comparison, we also display the electron velocity in the absence of self-energy correction (the gray lines in Fig. 7). The relative change in the renormalized velocity with respect to the bare velocity provides a measure of the interaction strength. In general, the electron-electron interaction increases the effective electron velocity similar to what has been seen in graphene. ${ }^{54}$ Our calculations show that the effect of the self-energy correction on the renormalized velocity becomes greater in gapped $\left(K_{1},-\right)$ and $\left(K_{2},+\right)$ subbands, except at very small wave vectors. Moreover, it is observed that while subbands with similar dispersions have an analogous renormalized velocity, the different magnitudes are related to the difference between their electron densities.

\section{CONCLUSION}

In this paper, we have studied the dynamical spin- and valley-dependent self-energy corrections to the electronic energy due to the electron-electron interaction within the $\mathrm{G}^{0} \mathrm{~W}$-RPA approximation for an n-type doped silicene at zero temperature. In particular, we have considered the interesting VSPM phase of silicene in the presence of an external exchange field. Our numerical results have shown that the real and imaginary parts of the self-energy may behave differently in distinct subbands, and some features of corresponding results for both 2D electron gas and graphene can be obtained in VSP silicene. Furthermore, the corresponding single-particle spectral function has been investigated, and it is seen that existence of the quasiparticle and plasmaron peaks and its specific behavior depends on the electron density of each state and also on the substrate. Our calculations have suggested that these peaks exhibit different broadenings and positions in different subbands. We have discussed this effect based on the fact that in the VSPM phase under an external exchange field, the individual subbands have different electron densities that can affect the spectral function. Moreover, we have calculated the renormalized velocity of four valley and spin-dependent states as a function of wave vector in the on-shell approximation. We have found that this many-body quantity, which can be experimentally measured, is an increasing function of wave vectors for the gapped subbands and a slowly decreasing function for the gapless subbands. Furthermore, we see that increasing the background dielectric constant decreases the renormalized velocity. We expect our findings to be potentially useful for interpreting relevant experimental results in silicene-like materials.

\section{ACKNOWLEDGMENTS}

B.T. acknowledges support from TUBITAK (Grant No. 117F125) and TUBA (Grant No. AD19).

\section{REFERENCES}

${ }^{1}$ K. S. Novoselov, A. K. Geim, S. Morozov, D. Jiang, M. Katsnelson, I. Grigorieva, S. Dubonos, and A. A. Firsov, Nature 438, 197 (2005).
${ }^{2}$ R. Mas-Balleste, C. Gomez-Navarro, J. Gomez-Herrero, and F. Zamora, Nanoscale 3, 20 (2011).

${ }^{3}$ A. Gupta, T. Sakthivel, and S. Seal, Prog. Mater. Sci. 73, 44 (2015).

${ }^{4}$ J. R. Schaibley, H. Yu, G. Clark, P. Rivera, J. S. Ross, K. L. Seyler, W. Yao, and X. Xu, Nat. Rev. Mater. 1, 16055 (2016).

${ }^{\mathbf{5}}$ M. Alidoust, M. Willatzen, and A.-P. Jauho, Phys. Rev. B 99, 125417 (2019).

${ }^{6}$ M. Alidoust, M. Willatzen, and A.-P. Jauho, Phys. Rev. B 98, 184505 (2018).

${ }^{7}$ M. Alidoust, M. Willatzen, and A.-P. Jauho, Phys. Rev. B 98, 085414 (2018).

${ }^{8}$ G. Huang, Z. Xing, and D. Xing, Appl. Phys. Lett. 106, 113107 (2015).

${ }^{9}$ S. Barua, K. Rajeev, and A. K. Gupta, J. Phys.: Condens. Matter 27, 015601 (2014).

${ }^{10}$ R. Zhang, J. Waters, A. K. Geim, and I. V. Grigorieva, Nat. Commun. 8, 15036 (2017).

${ }^{11}$ M. Houssa, A. Dimoulas, and A. Molle, J. Phys.: Condens. Matter 27, 253002 (2015).

${ }^{12}$ P. Vogt, P. De Padova, C. Quaresima, J. Avila, E. Frantzeskakis, M. C. Asensio, A. Resta, B. Ealet, and G. Le Lay, Phys. Rev. Lett. 108, 155501 (2012).

${ }^{13}$ L. Meng, Y. Wang, L. Zhang, S. Du, R. Wu, L. Li, Y. Zhang, G. Li, H. Zhou, W. A. Hofer et al., Nano Lett. 13, 685 (2013).

${ }^{14}$ A. Fleurence, R. Friedlein, T. Ozaki, H. Kawai, Y. Wang, and Y. Yamada-Takamura, Phys. Rev. Lett. 108, 245501 (2012).

${ }^{15}$ C.-C. Liu, H. Jiang, and Y. Yao, Phys. Rev. B 84, 195430 (2011).

${ }^{16}$ S. Dushenko, H. Ago, K. Kawahara, T. Tsuda, S. Kuwabata, T. Takenobu, T. Shinjo, Y. Ando, and M. Shiraishi, Phys. Rev. Lett. 116, 166102 (2016).

${ }^{17}$ B. T. Zhou, K. Taguchi, Y. Kawaguchi, Y. Tanaka, and K. Law, Commun. Phys. 2, 26 (2019).

${ }^{18}$ D. Xiao, W. Yao, and Q. Niu, Phys. Rev. Lett. 99, 236809 (2007).

${ }^{19}$ R. Beiranvand, H. Hamzehpour, and M. Alidoust, Phys. Rev. B 96, 161403 (2017).

${ }^{20}$ J. Mendes, O. A. Santos, L. Meireles, R. Lacerda, L. Vilela-Leão, F. Machado, R. Rodríguez-Suárez, A. Azevedo, and S. Rezende, Phys. Rev. Lett. 115, 226601 (2015).

${ }^{21}$ A. Avsar, J. Y. Tan, T. Taychatanapat, J. Balakrishnan, G. Koon, Y. Yeo, J. Lahiri, A. Carvalho, A. Rodin, E. O'Farrell et al., Nat. Commun. 5, 4875 (2014).

${ }^{22}$ A. Kara, H. Enriquez, A. P. Seitsonen, L. L. Y. Voon, S. Vizzini, B. Aufray, and H. Oughaddou, Surf. Sci. Rep. 67, 1 (2012).

${ }^{23}$ N. Drummond, V. Zolyomi, and V. Fal'Ko, Phys. Rev. B 85, 075423 (2012)

${ }^{24}$ B. Van Duppen, P. Vasilopoulos, and F. Peeters, Phys. Rev. B 90, 035142 (2014).

${ }^{25}$ Y. Ye, J. Xiao, H. Wang, Z. Ye, H. Zhu, M. Zhao, Y. Wang, J. Zhao, X. Yin, and X. Zhang, Nat. Nanotechnol. 11, 598 (2016).

${ }^{26}$ M. Ezawa, Phys. Rev. B 87, 155415 (2013).

${ }^{27}$ C.-C. Liu, W. Feng, and Y. Yao, Phys. Rev. Lett. 107, 076802 (2011).

${ }^{28} \mathrm{M}$. Tahir, A. Manchon, K. Sabeeh, and U. Schwingenschlögl, Appl. Phys. Lett. 102, 162412 (2013).

${ }^{\mathbf{2 9}} \mathrm{M}$. Tahir and U. Schwingenschlögl, Sci. Rep. 3, 1075 (2013).

${ }^{30}$ L. Tao, E. Cinquanta, D. Chiappe, C. Grazianetti, M. Fanciulli, M. Dubey, A. Molle, and D. Akinwande, Nat. Nanotechnol. 10, 227 (2015).

${ }^{31}$ A. Grüneis, C. Attaccalite, T. Pichler, V. Zabolotnyy, H. Shiozawa, S. Molodtsov, D. Inosov, A. Koitzsch, M. Knupfer, J. Schiessling, and R. Follath, Phys. Rev. Lett. 100, 037601 (2008).

${ }^{32}$ G. Cappellini, J. Furthmüller, E. Cadelano, and F. Bechstedt, Phys. Rev. B 87, 075203 (2013).

${ }^{33}$ S.-K. Mo, Nano Convergence 4, 6 (2017).

${ }^{34}$ P. Sessi, V. M. Silkin, I. A. Nechaev, T. Bathon, L. El-Kareh, E. V. Chulkov, P. M. Echenique, and M. Bode, Nat. Commun. 6, 8691 (2015).

${ }^{35}$ W. Peng, T. Xu, P. Diener, L. Biadala, M. Berthe, X. Pi, Y. Borensztein, A. Curcella, R. Bernard, G. Prevot, and B. Grandidier, ACS Nano 12, 4754 (2018).

${ }^{36}$ L. Feng, K. Yabuoshi, Y. Sugimoto, J. Onoda, M. Fukuda, and T. Ozaki, Phys. Rev. B 98, 195311 (2018).

${ }^{37}$ T. Kaloni, M. Tahir, and U. Schwingenschlögl, Sci. Rep. 3, 3192 (2013). 
${ }^{38}$ G. D. Mahan, Many-Particle Physics (Springer Science \& Business Media, 2013).

${ }^{39}$ L. Reining, Wiley Interdiscip. Rev. Comput. Mol. Sci. 8, e1344 (2018).

${ }^{40}$ A. Bostwick, T. Ohta, T. Seyller, K. Horn, and E. Rotenberg, Nat. Phys. 3, 36 (2007).

${ }^{41}$ S. Hüfner, R. Claessen, F. Reinert, T. Straub, V. Strocov, and P. Steiner, J. Electron. Spectrosc. Relat. Phenom. 100, 191 (1999).

${ }^{42}$ C. J. Tabert and E. J. Nicol, Phys. Rev. B 89, 195410 (2014).

${ }^{43}$ N. Dadkhah, T. Vazifehshenas, M. Farmanbar, and T. Salavati-fard, J. Appl. Phys. 125, 104302 (2019).

${ }^{44}$ C.-H. Wu, Results Phys. 11, 665 (2018).

${ }^{45} \mathrm{~V}$. Vargiamidis and P. Vasilopoulos, J. Appl. Phys. 117, 094305 (2015).

${ }^{46}$ L. Stille, C. J. Tabert, and E. J. Nicol, Phys. Rev. B 86, 195405 (2012).

${ }^{47}$ H. Bao, W. Liao, J. Guo, X. Yang, H. Zhao, and G. Zhou, J. Phys. D Appl. Phys. 48, 455306 (2015).

${ }^{48}$ R. Jalabert and S. D. Sarma, Phys. Rev. B 40, 9723 (1989).

${ }^{49}$ L. Hedin and S. Lundqvist, Solid State Physics (Academic Press, New York, 1969), Vol. 23

${ }^{50}$ A. Sabashvili, S. Östlund, and M. Granath, Phys. Rev. B 88, 085439 (2013).

${ }^{51}$ E. Hwang and S. D. Sarma, Phys. Rev. B 77, 081412 (2008).

${ }^{52}$ M. Mirzaei, T. Vazifehshenas, T. Salavati-fard, M. Farmanbar, and B. Tanatar,

Phys. Rev. B 98, 045429 (2018).
${ }^{53}$ A. Bostwick, F. Speck, T. Seyller, K. Horn, M. Polini, R. Asgari, A. H. MacDonald, and E. Rotenberg, Science 328, 999 (2010).

${ }^{54}$ S. D. Sarma and E. Hwang, Phys. Rev. B 87, 045425 (2013).

${ }^{55}$ J. J. Quinn and R. A. Ferrell, Phys. Rev. 112, 812 (1958).

${ }^{56}$ R. Sensarma, E. Hwang, and S. D. Sarma, Phys. Rev. B 84, 041408 (2011).

${ }^{57}$ C. L. Kane and E. J. Mele, Phys. Rev. Lett. 95, 226801 (2005).

${ }^{58}$ C. L. Kane and E. J. Mele, Phys. Rev. Lett. 95, 146802 (2005).

${ }^{\mathbf{5 9}} \mathrm{M}$. Ezawa, New J. Phys. 14, 033003 (2012).

${ }^{60} \mathrm{H}$. Haugen, D. Huertas-Hernando, and A. Brataas, Phys. Rev. B 77, 115406 (2008).

${ }^{61}$ M. Ezawa, Phys. Rev. Lett. 109, 055502 (2012).

${ }^{62}$ X. Li, J. T. Mullen, Z. Jin, K. M. Borysenko, M. B. Nardelli, and K. W. Kim, Phys. Rev. B 87, 115418 (2013).

${ }^{63}$ B. Bishnoi and B. Ghosh, RSC Adv. 3, 26153 (2013).

${ }^{64} \mathrm{G}$. Giuliani and G. Vignale, Quantum Theory of the Electron Liquid (Cambridge University Press, 2005).

${ }^{65}$ E. Hwang, B. Y.-K. Hu, and S. D. Sarma, Physica E 40, 1653 (2008).

${ }^{66}$ P. Pyatkovskiy, J. Phys.: Condens. Matter 21, 025506 (2008).

${ }^{67}$ A. Qaiumzadeh and R. Asgari, New J. Phys. 11, 095023 (2009).

${ }^{68} \mathrm{P}$. von Allmen, Phys. Rev. B 46, 13345 (1992). 